\title{
The Videofluorographic Swallowing Study: A Review of Literature
}

Umar Farooque ${ }^{1}$, Syed Adeel Hassan ${ }^{1}$, SM Ismail Shah² ${ }^{2}$ Saba Asif ${ }^{3}$, Muhammad Humayoun Rashid ${ }^{4}$, Abubakar Tauseef ${ }^{5}$

1. Internal Medicine, Dow University of Health Sciences, Karachi, Pakistan.

2. Internal Medicine, Ziauddin University, Karachi, Pakistan.

3. Internal Medicine, University at Buffalo, Buffalo, USA.

4. Internal Medicine, Nishtar Medical University, Multan, Pakistan.

5. Internal Medicine, Creighton University Medical Center, Omaha, USA.

\section{Corresponding author}

Umar Farooque, Internal Medicine, Dow University of Health Sciences, Karachi, Pakistan. E-mail address: umarfarooque65@gmail.com

Word count: 1700 


\begin{abstract}
The videofluorographic study is indicated for diseases potentially causing dysphagia and aspiration, such as gastrointestinal reflux disease, cancers of the oropharynx and esophagus, and certain neurologic diseases needing swallowing assessment. The study is designed to survey the anatomy and physiology of swallowing to diagnose any swallowing impairment and is standardized using scales such as the Modified Barium Swallow Impairment Profile (MBSImP). The procedure requires the patient to swallow a barium contrasted bolus which helps to visualize and obtain a real-time result. There are certain complications of the procedure including radiation exposure which requires necessary safety protocols to be followed.
\end{abstract}

\title{
Introduction \& Background
}

The videofluorographic swallowing study is an important initial diagnostic study for dysphagia as it helps to see the relative structural motion of the upper aerodigestive pathway in real-time with the motion of food bolus passing through it [1]. Aspiration can also be detected with the help of the swallowing study [2]. This study helps the clinicians in assessing the effects on swallowing physiology with different texture and size of the bolus [3]. Patients with dysphagia are evaluated with videfluorographic swallowing study to assess the degree of swallowing impairment. The interpretation of the study requires the expertise of radiologists and speech-language pathologists (SLPS) [4].

\section{Review}

\section{Procedure}

The procedure of swallowing study involves giving the patient barium contrast impregnated food or liquid to ingest. Barium helps in visualizing the movement of the bolus in real-time through the upper aerodigestive pathway. The patient may be directed to sit in a chair or to stand on a platform. A secure seat is usually used for children and infants. As the patient swallows the provided barium impregnated food or drink, the $\mathrm{x}$ ray camera is moved closer to the patient to obtain a real-time result of the swallowing process. The videoradiographic images of the barium impregnated bolus are obtained sequentially as the bolus moves through the oral cavity, pharyngeal cavity, and esophagus. Different textures and volumes of liquid and food are administered; the impression of the degree of swallowing impairment is then made based on radiographic images obtained [4]. These clinical impressions include the evaluation of timing and coordination of the events involved in the process of swallowing. Recommendations regarding the type of diet, oral vs non-oral diet, and referrals are made using the results of the videofluorographic swallowing study [5]. 


\section{Indications}

Different conditions like gastroesophageal reflux disease, cancers of oropharynx and esophagus, stroke, and numerous neurological conditions can result in dysphagia or difficulty in swallowing [6]. Dysphagia is also encountered in more than two-thirds of the patient requiring intubation longer than forty-eight hours [7]. Multiple complications like aspiration and decreased food and liquid intake can occur as a result of dysphagia [8]. In such cases, a videofluorographic swallowing study is indicated to assess the degree of swallowing impairment, delay in pharyngeal swallowing, presence of ingestate in the pharyngeal cavity after swallowing, nasopharyngeal regurgitation, and the presence of aspiration in the patient [9]. Moreover, this study is indicated when there is a need to assess the anatomy and physiology of swallowing, integrity of airway pathways, therapeutic techniques for laryngeal and oropharyngeal disorders, and to obtain information that might help in making decisions regarding swallow safety [10].

\section{Potential diagnosis}

The swallowing study is useful in assessing any swallowing impairment. The results will indicate whether there is a need for a limited diet or a normal regular diet. The decision of opting for non-oral feeding is also dependent on the results of this study.

Occasionally, a strictly limited diet is recommended in patients on a regular normal diet. Conversely, the normal regular diet may be recommended in patients on a strictly limited diet [11].

\section{Normal and critical findings}

The results of videofluoroscopic swallowing study are dependent on different factors like the expertise of radiologists interpreting the result. There can be substantial inter-rater variability. Therefore, different strategies are adopted to standardize the results of the study. These involve using certain scales like "Penetration aspiration scale", "Dysphagia severity rating scale" and "Modified Barium Swallow Impairment Profile (MBSImP)". Since these scales help in producing a digital scale result; they help in standardizing the test. The penetration aspiration scale has a score range from one to eight. One represents no amount of penetration or aspiration, two to five represents an increasing amount of penetration, and six to eight represents an increasing amount of aspiration on the scale. The dysphagia severity rating scale has a scale range from zero to six. Zero represents normal functioning and six represents severe dysphagia [10]. The MBSImP assesses 17 physiological components involved in swallowing [12]. These include proper closure of the lips, control of the tongue during bolus hold, preparation of the 
bolus, transport of the bolus, oral residue, pharyngeal swallow initiation, the elevation of soft palate and larynx, anterior hyoid excursion, epiglottic movement, closure of the laryngeal vestibule, pharyngeal stripping wave, contraction of the pharynx, pharyngoesophageal opening, retraction of the tongue base, pharyngeal residue, and esophageal clearance. The final score is based on the evaluation of these physiological components and will indicate what degree of dysphagia or swallowing impairment is present [13].

\section{Interfering factors}

The patient is usually asked to remove any spectacles, jewelry, or removable dental appliances. Furthermore, any metal object that might interfere with the imaging is also asked to remove. Ideally, the patient should be asked to change into a gown provided by the imaging facility [5].

\section{Complications}

The patient undergoing this swallowing study may feel nauseous or vomit nearly 30 minutes after the procedure. The occurrence of hypersensitivity reactions is a rare possibility. Constipation may also occur which can be managed with over the counter laxatives. Increased water intake is recommended for constipation. The patient may also pass white-colored stools for a day or two. This is due to barium in the stools [14].

\section{Patient safety and education}

It is very important to question the patient about any past incident of an allergic reaction to barium. If the patient is allergic to barium, an alternate diagnostic study can be chosen to assess the condition. If the patient is breastfeeding or pregnant, the healthcare provider should be informed. The decision to follow through with the procedure is then based on the risk assessment and benefits of the study. The patient should be educated that this is a painless procedure and barium does not have any harmful effects on the body. They should also be warned about potential side effects like constipation and white-colored stools [6]. Radiation safety is a very important issue. The target should be to achieve the imaging study with the least amount of radiation exposure necessary. Moreover, the SLPs or radiologists performing these procedures should follow the protocols and wear appropriate shielding like lead-aprons, thyroidshields, lead-gloves, and eye shields to protect themselves. Dosimetry badges are also required to keep track of the radiation exposure in the SLPs and radiologists [15].

\section{Clinical significance}


Swallowing is a complex physiological phenomenon that has both sensory and motor components. The sensory component is responsible for initiating the motor response. This test indirectly assesses both components of swallowing when performed by trained personnel [16]. Patients with a stroke can also have dysphagia depending on the region of the brain affected. In such cases, aspiration is a life-threatening risk that needs to be avoided. This swallowing study is useful in assessing the integrity of the swallowing mechanism. The results of the study will help in choosing the right kind of diet (normal regular diet or limited diet) and the decision of opting oral vs nonoral diet [6].

\section{Conclusions}

The videofluorographic swallowing assessment provides a dynamic and accurate observation of impairments in the natural swallowing processes. An optimum treatment plan can be generated for the patient based on the observational study using a real-time assessment. However, radiation exposure during the procedure should be minimized, while ensuring maximum yield of results.

\section{References}

1. Robbins J, Coyle J, Rosenbek J, Roecker E, Wood J: Differentiation of normal and abnormal airway protection during swallowing using the penetrationaspiration scale. Dysphagia. 1999, 14:228-32. 10.1007/PL00009610

2. Dodds WJ, Logemann JA, Stewart ET: Radiologic assessment of abnormal oral and pharyngeal phases of swallowing. American Journal of Roentgenology. 1990, 154:965-74. 10.2214/ajr.154.5.2108570

3. Logemann JA: Behavioral management for oropharyngeal dysphagia. Folia Phoniatr Logop. 1999, 51:199-212. 10.1159/000021497

4. Matsuo K, Palmer JB: Video fluoroscopic techniques for the study of oral food processing. Curr Opin Food Sci. 2016, 9:1-10. 10.1016/i.cofs.2016.03.004

5. Martin-Harris B, Logemann JA, McMahon S, Schleicher M, Sandidge J : Clinical utility of the modified barium swallow. Dysphagia. 2000, 15:13641. 10.1007/s004550010015

6. Peterson R: Modified barium swallow for evaluation of dysphagia. Radiol Technol. 2018, 89:257-75.

7. Skoretz SA, Flowers HL, Martino R: The incidence of dysphagia following endotracheal intubation: a systematic review. Chest. 2010, 137:665-

73. 10.1378/chest.09-1823

8. Palmer JB, Drennan JC, Baba M: Evaluation and treatment of swallowing impairments. Am Fam Physician. 2000, 61:2453-62.

9. Logemann JA, Gensler G, Robbins J, et al.: A randomized study of three interventions for aspiration of thin liquids in patients with dementia or Parkinson's disease. J Speech Lang Hear Res. 2008, 51:173-83. 10.1044/1092$\underline{4388(2008 / 013)}$ 
10. How to perform video-fluoroscopic swallowing studies | Goyal and Shaker GI Motility Online. (2006). Accessed: July 25, 2020: https://www.nature.com/gimo/contents/pt1/full/gimo95.html.

11. Kim SB, Lee SJ, Lee KW, Lee JH, Kim DW: Usefulness of early videofluoroscopic swallowing study in acute stroke patients with dysphagia. Ann Rehabil Med. 2018, 42:42-51. 10.5535/arm.2018.42.1.42

12. Martin-Harris B, Brodsky MB, Michel Y, et al.: MBS measurement tool for swallow impairment-MBSImp: establishing a standard. Dysphagia. 2008, 23:392405. 10.1007/s00455-008-9185-9

13. Kooi AH, Boo JP, Ng SY, et al.: The modified barium swallow impairment profile as a predictor of clinical outcomes of admission for pneumonia or choking in dysphagic patients with Parkinson's disease. Dysphagia. 2019, 34:896903. 10.1007/s00455-019-09986-9

14. Chen A, Tafti D, Tuma F: From Barium swallow. In StatPearls. StatPearls Publishing, Treasure Island (FL); 2020.

15. Kelchner LN: Radiation safety during the videofluoroscopic swallow study: the adult exam. Perspectives on Swallowing and Swallowing Disorders (Dysphagia). 2004, 13:24-28. 10.1044/sasd13.3.24

16. Martin-Harris B, Jones B: The videofluorographic swallowing study. Phys Med Rehabil Clin N Am. 2008, 19:769-85. 10.1016/j.pmr.2008.06.004 